\title{
MiR-125b is a Potential Protector of Arteriosclerosis Occlusive: Restricting the Proliferation and Migration of Vascular Smooth Muscle Cells via Decreasing the Level of AAMP and SRF.
}

\section{Xiaogao Wang}

The First Affiliated Hospital of Bengbu Medical College

\section{Shiyuan Chen}

The First Affiliated Hospital of Bengbu Medical College

Yong Gao ( $\sim$ yonggao03345@163.com )

The First Affiliated Hospital of Bengbu Medical College https://orcid.org/0000-0001-5448-9460

\section{Chaowen Yu}

The First Affiliated Hospital of Bengbu Medical College

\section{Zhonglin Nie}

The First Affiliated Hospital of Bengbu Medical College

\section{Yong Sun}

The First Affiliated Hospital of Bengbu Medical College

\section{Wenbo Tang}

The First Affiliated Hospital of Bengbu Medical College

\section{Zeyu Guan}

The First Affiliated Hosptal of Bengbu Medical College

Research article

Keywords: arteriosclerosis occlusive (ASO), miR-125b, vascular smooth muscle cells (VSMCs)

Posted Date: April 30th, 2020

DOI: https://doi.org/10.21203/rs.3.rs-23946/v1

License: (c) (i) This work is licensed under a Creative Commons Attribution 4.0 International License. Read Full License 


\section{Abstract}

Arteriosclerosis occlusive (ASO) is a manifestation of atherosclerosis (AS), and it is one of the main causes of lower limb ischemic necrosis in the elderly. Excessive proliferation and migration of vascular smooth muscle cells is one of the main causes of ASO, but the mechanism is unclear. MiR-125b is a tumor suppressor, which can inhibit the proliferation and migration in various kinds of tumors. At the same time, we find miR-125b is the most obviously down-regulated microRNA in ASO. Therefore, we explore the role of miR-125b in ASO. This study shows that up-regulated miR-125b inhibits the proliferation of vascular smooth muscle cells through CCK8 assay and Brdu assay. Meantime, the upregulation of miR-125b restricts the migration of vascular smooth muscle cells through trans-well assay and wound healing assay. Furthermore, this study finds that up-regulated miR-125b inhibits the expression level of angio-associated migratory cell protein (AAMP) and serum response factor (SRF), which may be an important way for miR-125b to regulate the proliferation and metastasis of smooth muscle cells.

\section{Introduction}

Atherosclerosis (AS) mainly refers to the deposition of lipids on blood vessel walls, which caused by abnormal metabolism [1]. Lower extremity arteriosclerosis occlusive (ASO) is the performance of AS in the lower limbs. To our knowledge, about $80 \%$ of the people, over 60 years old, undergo the ASO. The ASO can be rapidly relieved by interventional therapy. However, the 1-year-recurrence rate of stenosis up to $30 \%$ 50\%, and about $12 \%$ of which who have severe lower limb ischemic necrosis even meet amputation[2]. So, it is a necessity to find out the molecular mechanisms in ASO process, which might provide strategy for clinical treatments.

ASO is originally initiated by the accumulation of apolipoproteins in endothelium, which come from the abnormal metabolism of glucose and lipid [1, 3]. Following, the accumulation of apolipoproteins leads a series of intracellular reactions, which eventually make vascular endothelial damage. Then, monocyte macrophages-induced inflammatory response appears [4]. Inflammatory cells and inflammatory factors regulate the function of vascular smooth muscle cells (VSMCs), including cell proliferation, migration, differentiation and matrix secretion [4]. VSMCs, as the main cellular component of the middle layer of the arteries, its excessive proliferation and migration play pivotal roles in ASO and restenosis after interventional treatment [5]. Therefore, the prevention and relief of dysfunction of VSMC are important.

Recent studies have shown that microRNAs (miR) are involved in the occurrence and progression of cardiovascular diseases. For example, miR-124 promotes the degradation of TNF-a protein, which enhances inflammatory response and endothelial damage, and promotes the progression of ASO [6]. MiR-146a makes hyperproliferation in VSMCs and indirectly promotes the occurrence of ASO by upregulating the expression of BAX [7]. MiR-221 can significantly strengthen the migration and proliferation abilities of VSMCs [8]. However, there are microRNAs that inhibit ASO. MiR-143/145 regulate the expression of mammalian transcription factors and myocardinto, by serum response factors (SRF), to 
promote the differentiation of SVMC and to inhibit its proliferation, by which MiR-143/145 prevent VSMCs from further damage $[9,10]$. In addition, miR-21, miR-143and miR-221 are involved in the pathogenesis of ASO [11, 12]. Nonetheless, those discovered microRNAs have made few contributions to the clinical treatment of ASO.

MiR-125b is down-regulated in a variety of tumor cells, including hepatocellular carcinoma, breast cancer, ovarian tumors, bladder cancer and leukemia [13-17]. When miR-125b expression is down-regulated, these cancer cells have enhanced proliferation and invasion capabilities. In ASO, miR-125b is one of the microRNAs with the most significant change in expression level [6]. Some research results suggest that miR-125b can inhibit the proliferation and migration of VSMCs. Meantime, some studies have shown that miR-125b can participate in the regulation of SRF, thereby promoting cellular migration [6]. However, in ASO, the molecular regulation mechanism of miR-125b on them is unclear. Therefore, we carry out experiments on how miR-125b regulates the arterial endothelial cell damage, and want to provide new strategies for clinical treatment of ASO.

In summary, our experiments obtain the following conclusions: 1. PDFG-BB can inhibit the expression of microNRA-125b; 2 . Overexpression of miN-125b can inhibit the proliferation and migration of VSMC; 3 . miR-125b can down-regulate SRF and AAMP to inhibit migration and proliferation of VSMC.

\section{Methods And Materials}

\section{Cell Culture}

Aortic vascular smooth muscle cells (A10 cell) are gained from the cell bank of Chinese academy of science in October 2017 with STR matching analysis. A10 cells are cultured in DMEM (Gibco, USA) with $10 \%$ fetal calf serum, 100 units $/ \mathrm{mL}$ penicillin and $100 \mathrm{units} / \mathrm{mL}$ streptomycin.

\section{Quantification Real Time Pcr (qrt-pcr)}

Cells with different treatments are collected, and the total RNA extraction is performed by Trizol RNA solation system (Invitrogen, USA). Then the reverse transcription of cDNA is performed by PrimeScript RT Reagent Kit (TaKaRa, China). The mRNA level is analyzed through a 7500 Fast $^{\text {TM }}$ System (Applied Biosystems, USA), with the help from the Sensi Mix SYBR Kit (Bio-Rad, USA). " $=2^{-} \triangle \triangle \mathrm{C}$ " is applied in calculating the absolute expression of mRNA, which normalized to GAPDH.

\section{Western Blot Assay}

Cells with different treatments are harvested by cytology brush, followed by iced PBS washing, and lysed with RIPA lysis buffer (Sigma, USA), in which containing phosphorylase and protease inhibitor mixture (Thermo, USA). Then, total protein level is quantified by the BCA assay. Finally, specific gels ware 
prepared for electrophoresis. A PVDF membrane is applied in immunoblotting after electrophoresis. Secondary antibody incubation was performed in TBST solution with $5 \%$ skim milk, which contains different antibodies (antibodies against GAPDH, SRF and AAMP are purchased from Abcam (1:1000)).

\section{Immunofluorescence}

Briefly, A10 cells are seeded in 24-well plates for $24 \mathrm{~h}$, and then fixed by $4 \%$ paraformaldehyde, followed by permeabilization within $0.5 \%$ Triton $X-100$, and blocking within $5 \%$ bovine serum albumin (BSA, Sigma). Samples are incubated with primary antibody (SRF, 1:200; AAMP, 1:200) overnight at $4{ }^{\circ} \mathrm{C}$. Subsequently, it washed by PBS, incubated with secondary antibodies for $1 \mathrm{~h}$ in room temperature before being washed again. Finally, nuclei are stained with $15 \mu \mathrm{L}$ DAPI (Sigma, USA) before detected by fluorescence microscope (Carl Zeiss, Germany).

\section{Mimic Transfection}

A10 cells are transplanted into 6 wells plates for $24 \mathrm{~h}$, and then cells were transfected with mimic-125b (GenePharma, Shanghai, China) for $24 \mathrm{~h}$ with Lipofectamine 3000 reagent (Invitrogen, USA) and OptiMEM (Life Technologies, USA), according to the manufacturer's instructions for gaining the best transfection efficiency.

\section{Cellular Proliferation Assay}

Cell proliferation was measured by CCK8 assay and Brdu assay. For CCK8 assay, cell proliferation is quantified by standard curve $\left(0.1,0.2,0.4,0.8,1.0,1.5,2.0,3.0 \times 10^{4}\right)$ cells are detected optical density (OD) via cell counting kit-8 (Japan) after $24 \mathrm{~h}$ transplanted into 96-wells plates, and then fit linear standard curve between log [cell quantity] and OD. For Brdu assay, cells are seeded in 24-wells plates for $24 \mathrm{~h}$, which followed by different treatments. Then, cellular proliferation was performed with BeyoClick ${ }^{\mathrm{TM}}$ EDU-647 system (Ruanbotio, Shanghai). The absorbance was measured in $450 \mathrm{~nm}$.

\section{Migration and Invasion Experiments}

For transwell assay, $5.0 \times 10^{4}$ cells with different treatments are transplanted into transwell plates (24well, $8.0 \mu \mathrm{m}$, Corning Incorporated, Corning, NY, USA) with $5 \sim 10 \%$ gradient of fetal calf serum for $48 \mathrm{~h}$. And the detection procedure was performed with crystal violet and microscope. Quantification of migrated cells were calculated by microscope. For wound healing assay, cells are seeded at least $80 \%$ fusion in 6-well plates, and scratched by 200 ul pipette tip, then wash with PBS to remove shed cells for extra $48 \mathrm{~h}$ culture. Scratch distance is also calculated by microscope.

\section{Statistics}


All experimental data are presented as the means \pm SD. Statistical Package for the Social Sciences version 20.0 (SPSS Inc., USA) is used for statistical analyses. ANOVA, paired t-test, Chi-square () test and nonparametric test (Mann Whitney $U$ ) for statistical analysis of different situations. Statistical significance is considered when $p<0.05\left({ }^{\star} p<0.05 ;{ }^{* *} p<0.01\right.$; $\left.{ }^{* \star} p<0.001\right)$. All histograms and curves are constructed with GraphPad Prism 6 (GraphPad Software, La Jolla, CA, USA).

\section{Results}

\section{PDGF-BB plays a negative role in regulation of miR-125b in VSMCs.}

Platelet-derived growth factor (PDGF) is a prominent inducer of vascular cell dysfunction, especially the PDGF-BB, which can trigger a variety of biological effects. By activating intracellular signal transduction pathways, PDGF-BB plays an important role in dysfunction of VSMCs, such as migration and proliferation. Therefore, PDGF-BB is used as an inducer in this study. In this paper, we explore the role of PDGF-BB in regulation of miR-125b. As the Fig. 1A showing, we set four groups: negative control group (NC, treated with medium), Mimic-125b group (treated with mimic-125b-loaded liposomes), PDGF-BB group (treated with $20 \mathrm{ng} / \mathrm{mL}$ DPGF-BB) and Mimic-125b + PDGF group (treated with mimic-125b-loaded liposomes and $20 \mathrm{ng} / \mathrm{mL}$ DPGF-BB). The experiment results show that the transfection of mimic-125b increases the level of miR-125b about 20 -fold as comparing with NC (Fig. $1 \mathrm{~A}, \mathrm{p}<0.001$ ), while the treatment of PDGF-BB decreases the miR-125b level about $80 \%$ in VSMCs (Fig. 1A, $p<0.001$ ). Interestingly, $20 \mathrm{ng} / \mathrm{mL}$ treatment of PDGF-BB abrogates the Mimic-125b-loaded liposomes-induced overexpression of miR-125b, which even down-regulates the level of miR-125b to fall below the normal level (60\% down-regulation, compared with NC) (Fig. 1A, $p<0.001)$. In addition, we find that Mimic-125bloaded liposomes exactly reverses PDGF-BB-induced down-regulation of miR-125b (Fig. 1b, $p<0.05$ ).

\section{MiR-125b restricts the cellular proliferation of VSMCs.}

In order to exploring the role of miR-125b in regulation of cellular proliferation in VSMCs, we perform CCK assay and Brdu assay. Basing on the above results, we set two groups: NC group (treated with NC-loaded liposomes) and Mimic-125 group (treated with mimic-125b-loaded liposomes). As the results showing, Mimic-125b group holds lower OD value in $450 \mathrm{~nm}$, which is about half of it in NC group (Fig. 2A and B, $p$ $<0.001$ ). In other words, the up-regulation of miR-125b inhibits the cellular proliferation about $50 \%$ in VSMCs. For verifying the results, we perform Brdu experiment. As the Fig. $2 \mathrm{C}$ and $\mathrm{D}$ showing, transfection of mimic-125b makes nothing in cellular proliferation within $24 \mathrm{~h}$, while it significantly inhibits the OD value in $450 \mathrm{~nm}$ at day 4 (about $40 \%, \mathrm{p}<0.001$ ). It means the increasing of miR-125b can inhibit the cellular proliferation in VSMCs.

\section{MiR-125b inhibits the cellular migration and invasion of VSMCs.}

MiR-125b is an inhibitor in cellular proliferation and invasion in tumors. So, we attempt to find out the relationship between miR-125 and cellular migration in VSMCs. Firstly, we treat the VSMCs with mimic125b-loaded liposomes or NC-loaded liposomes for $24 \mathrm{~h}$, which followed by transplantation in trans-well 
for another $48 \mathrm{~h}$. Our three respective repeated data show that Mimic-125b group holds about 450 migrated cells in field, while NC group holds about 650 migrated cells in field (Fig. 3B). After normalization, we find migrated cells of Mimic-125b group are about $70 \%$ of which in NC group (Fig. $3 \mathrm{C}$ ). Furthermore, our experiments show that up-regulation of miR-125b decreases the migration ability about $30 \%$ in VSMCs $(p<0.05$, Fig. 3B-D). In order to verifying the above results, we apply the wound healing assay for testing migration capability. VSMCs are seeded in 6-well plates, which are treated with NCloaded liposomes or mimic-125b-loaded liposomes for $24 \mathrm{~h}$. Then refresh the medium for another $48 \mathrm{~h}$ culture after being scratched by 200 ul pipette tip. As the Fig. 4A showing, there are few differences in NC group and mimic-125b group at first $24 \mathrm{~h}$. However, at the second $24 \mathrm{~h}$, we find that the borders of the left and right parts almost coincide in NC group, while the gaps are still visible to the naked eye in Mimic$125 \mathrm{~b}$ group. Through measuring the distance between the two borders of right and left parts, we make digital transformation. Just as the Fig. $4 \mathrm{~B}$ showing, the distance between the two borders is about $750 \mu \mathrm{m}$ in NC group at second $24 \mathrm{~h}$, while it is about $700 \mu \mathrm{m}$ in mimic-125b group. After normalization, we find the migration ability in mimic-125b group is not more than $90 \%$ of which in NC group $(p<0.05$, Fig. 4C). In other words, the up-regulation of miR-125b inhibits the migration in VSMCs, about $12 \%(p<$ 0.001, Fig. 4D).

\section{AAMP and SRF are the targets of miR-125b for regulating the metastasis and proliferation in VSMCs.}

Angio-associated migratory cell protein (AAMP) and serum response factor (SRF) are directly involved in cellular migration and proliferation, which plays pivotal role in ASO. So, we explore the inner relationship between the miR-125b and AAMP\&SRF. Firstly, we use mimic-125-loaded liposomes or mimic-125b inhibitor-loaded liposomes (mimic-125bi-loaded liposomes) to make miR-125b up- or down- regulated cells. Just as Fig. 5 showing, Mimic-125b group holds more than 2-fold increasing of miR-125b when compares with NC group $(p<0.001)$. And the Mimic-125bi group almost doesn't have the expression of miR-125b, as comparing with NC group $(p<0.001$, Fig. 5$)$. Following, we test the protein level of AAMP and SRF after treatment of mimic-125b or mimic-125bi. Our results show that AAMP and SRF are significantly down-regulated in Mimic-125b group (AAMP: about 60\%, SRF: 40\%), while which are upregulated in Mimic-125bi group (AAMP: 1.25-fold, SRF: about 1.5-fold) (Fig. 6A and B). However, in the IF assay, the mimic-125b-induced down-regulation of AAMP and SRF is not conspicuous, while the mimic125bi-induced up-regulation of AAMP and SRF is significant. In summary, up-regulation of miR-125b inhibits the expression level of AAMP and SRF.

\section{Discussion}

Lower extremity arteriosclerotic occlusive (ASO) is induced by atherosclerotic thrombosis, which causes narrowing or occlusion of the lower extremity arteries. And the persistent ischemia eventually leads to tissue necrosis. Most people with ASO don't have intermittent claudication symptoms, and only $10 \sim 30 \%$ of which show these symptoms. In the preclinical stage of ASO, it's different to make early diagnosis through clinical routine screening. This makes misunderstanding that ASO is an uncommon disease in people under 55 years old. However, with the increasing of the age, the prevalence of ASO is dramatically 
increased, which attacks about $8-10 \%$ of people over 65 years old and about $20 \%$ of people over 80 years old[18]. Therefore, early diagnosis provides early treatment opportunities for relieving lower limb arterial ischemia caused by ASO.

Vascular smooth muscle cells (VSMCs) are the major cellular components that make up the middle layer of the arteries and are involved in the process of vascular diseases. At present, it is believed that the excessive proliferation and migration of VSMCs is one of the main causes of ASO, and it is also the main cause of restenosis after interventional treatment [5]. Recently, more and more evidences imply that microRNA can regulate the function of VSMCs, endothelial cells and macrophages, thereby affecting the pathogenesis and progression of ASO[19]. Previous studies have confirmed that miR-21, miR-143 and miR-221 are involved in the pathogenesis of ASO [11, 12]. For example, miR-21 can regulate the proliferation and migration of VSMCs by targeting TPM1, thereby affecting the pathogenesis and progression of ASO. The deletion of miR-1298 is related to the $\mathrm{CpG}$ hypermethylation of ASO, and the down-regulation of miR-1298 regulates the proliferation and migration of VSMCs by directly targeting connexin-43, which plays a role in angiogenesis [20]. MiR-125b is one of the microRNAs which are the significantly down-regulated in ASO. Some research results suggest that miR-125b can inhibit the proliferation and migration of VSMCs, but the mechanism is unclear. In this study, we find that upregulation of PDGF-BB in VSMCs can significantly inhibit the expression level of miR-125b (Fig. $1 \mathrm{~A}, \mathrm{p}<$ 0.001). At the same time, the decreasing of miR-125b leads to significant up-regulation of AAMP and SRF proteins in VSMCs (Fig. 6A-D). In the following experiments, we find that VSMCs in NC group, with lower expression level of miR-125b, holds stronger migration and invasion ability, as comparing with the cells in experimental group ( $p<0.05$, Figs. 3 and 4 ). On the other hand, transfection of mimic-125 increases the level of miR-125b in VSMCs ( $p<0.05$, Fig. 1b). And the up-regulated protein level of miR-125b significantly restricts the growth and proliferation capability of VSMCs (about $50 \%, p<0.001$, Fig. 2). Meantime, the up-regulation of miR-125b significantly inhibits the migration and invasion of VSMCs (through trans-well assay and wound healing assay) ( $p<0.05$, Figs. 3 and 4$)$. Furthermore, the upregulation of miR-125b significantly down-regulates the level of AAMP and SRF in VSMCs (Fig. 6).

\section{Conclusion}

Therefore, for the above data, we can conclude that miR-125b may inhibit the proliferation and migration of vascular smooth muscle cells by inhibiting the expression of AAMP and SRF. In other words, the decreasing of miR-125b may be an inducer of ASO. Therefore, observing the expression of miR-125b probably can provide evidence for predicting the occurrence of ASO in the preclinical stage. And the increasing of miR-125b may provide a therapeutic strategy for preventing the progression of ASO.

\section{Declarations}

\section{Funding}


This work was supported by the Major Natural Science Project of Universities in Anhui Province (no. KJ2016SD38), the Key Project of Natural Science Foundation of Bengbu Medical College in Anhui Province (no. BYKY1862ZD) and the Scientific and technological research project of Anhui Province (no.201904a07020020).

\section{Authors' contributions}

WX, CS: creating database. YC: performed statistical analysis. WX, CS: performed histological analysis and interpretation of the results. WX, CS, YC, NZ: obtained informed consent, performed surgery, checked manuscript. SY, TW, GZ: performed histological assessment. GY: designing the study, statistical assessment. All authors read and approved the final manuscript.

\section{Conflict of Interest}

The authors declared no conflict of interest.

\section{Consent for publication}

Informed consent given by the patients enrolled in the current study was obtained at the time of surgery.

\section{Ethics approval and consent to participate}

The current retrospective study was approved by the Ethics Committee at the Osaka Medical College and conducted in accordance with the Declaration of Helsinki.

\section{References}

1. Howard MD, Hood ED, Zern B, Shuvaev VV, Grosser T, Muzykantov VR. Nanocarriers for vascular delivery of anti-inflammatory agents. Annu Rev Pharmacol Toxicol. 2014;54:205-26.

2. Protogerou AD, Papadogiannis D, Safar ME. Letter by Protogerou et al regarding article, "Mortality and vascular morbidity in older adults with asymptomatic versus symptomatic peripheral artery disease". Circulation. 2010;121:e455.

3. Johnson RC, Leopold JA, Loscalzo J. Vascular calcification: pathobiological mechanisms and clinical implications. Circulation research. 2006;99:1044-59.

4. Furukawa K, Abumiya T, Sakai K, Hirano M, Osanai T, Shichinohe H, Nakayama N, Kazumata K, Hida $\mathrm{K}$, Houkin K. Increased Blood Viscosity in Ischemic Stroke Patients with Small Artery Occlusion Measured by an Electromagnetic Spinning Sphere Viscometer. Journal of stroke cerebrovascular diseases: the official journal of National Stroke Association. 2016;25:2762-9.

5. Doran AC, Meller N, McNamara CA. Role of smooth muscle cells in the initiation and early progression of atherosclerosis, Arteriosclerosis, thrombosis, and vascular biology, 28 (2008) 812819. 
6. Chen Z, Wang M, Huang K, He Q, Li H, Chang G. MicroRNA-125b Affects Vascular Smooth Muscle Cell Function by Targeting Serum Response Factor, Cellular physiology and biochemistry: international journal of experimental cellular physiology, biochemistry, and pharmacology, 46 (2018) 1566-1580.

7. Kumar S, Kim CW, Simmons RD, Jo H. Role of flow-sensitive microRNAs in endothelial dysfunction and atherosclerosis: mechanosensitive athero-miRs, Arteriosclerosis, thrombosis, and vascular biology, 34 (2014) 2206-2216.

8. Warnecke-Eberz U, Chon SH, Holscher AH, Drebber U, Bollschweiler E. Exosomal onco-miRs from serum of patients with adenocarcinoma of the esophagus: comparison of miRNA profiles of exosomes and matching tumor. Tumour biology: the journal of the International Society for Oncodevelopmental Biology Medicine. 2015;36:4643-53.

9. Briley-Saebo KC, Shaw PX, Mulder WJ, Choi SH, Vucic E, Aguinaldo JG, Witztum JL, Fuster V, Tsimikas S, Fayad ZA. Targeted molecular probes for imaging atherosclerotic lesions with magnetic resonance using antibodies that recognize oxidation-specific epitopes. Circulation. 2008;117:320615.

10. Peng H, Zhong M, Zhao W, Wang C, Zhang J, Liu X, Li Y, Paudel SD, Wang Q, Lou T. Urinary miR-29 correlates with albuminuria and carotid intima-media thickness in type 2 diabetes patients. PloS one. 2013;8:e82607.

11. Boucher JM, Peterson SM, Urs S, Zhang C, Liaw L. The miR-143/145 cluster is a novel transcriptional target of Jagged-1/Notch signaling in vascular smooth muscle cells. J Biol Chem. 2011;286:2831221.

12. Liu X, Cheng Y, Yang J, Xu L, Zhang C. Cell-specific effects of miR-221/222 in vessels: molecular mechanism and therapeutic application. J Mol Cell Cardiol. 2012;52:245-55.

13. Chapiro E, Russell LJ, Struski S, Cave H, Radford-Weiss I, Valle VD, Lachenaud J, Brousset P, Bernard OA, Harrison CJ, Nguyen-Khac F. A new recurrent translocation t(11;14)(q24;q32) involving IGH@ and miR-125b-1 in B-cell progenitor acute lymphoblastic leukemia. Leukemia. 2010;24:1362-4.

14. Guan Y, Yao H, Zheng Z, Qiu G, Sun K. MiR-125b targets BCL3 and suppresses ovarian cancer proliferation. International journal of cancer. 2011;128:2274-83.

15. Hirsch HA, Iliopoulos D, Joshi A, Zhang Y, Jaeger SA, Bulyk M, Tsichlis PN, Shirley Liu X, Struhl K. A transcriptional signature and common gene networks link cancer with lipid metabolism and diverse human diseases. Cancer cell. 2010;17:348-61.

16. Huang L, Luo J, Cai Q, Pan Q, Zeng H, Guo Z, Dong W, Huang J, Lin T. MicroRNA-125b suppresses the development of bladder cancer by targeting E2F3. International journal of cancer. 2011;128:175869.

17. Rajabi H, Jin C, Ahmad R, McClary C, Joshi MD, Kufe D. MUCIN 1 ONCOPROTEIN EXPRESSION IS SUPPRESSED BY THE miR-125b ONCOMIR. Genes cancer. 2010;1:62-8.

18. Malgor RD, Alahdab F, Elraiyah TA, Rizvi AZ, Lane MA, Prokop LJ, Phung OJ, Farah W, Montori VM, Conte MS, Murad MH, A systematic review of treatment of intermittent claudication in the lower 
extremities, Journal of vascular surgery, 61 (2015) 54 s-73 s.

19. Siasos G, Kollia C, Tsigkou V, Basdra EK, Lymperi M, Oikonomou E, Kokkou E, Korompelis P, Papavassiliou AG. MicroRNAs: Novel diagnostic and prognostic biomarkers in atherosclerosis, Current topics in medicinal chemistry, 13 (2013) 1503-1517.

20. Hu W, Wang M, Yin H, Yao C, He Q, Yin L, Zhang C, Li W, Chang G, Wang S. MicroRNA-1298 is regulated by DNA methylation and affects vascular smooth muscle cell function by targeting connexin 43. Cardiovascular research. 2015;107:534-45.

Figures
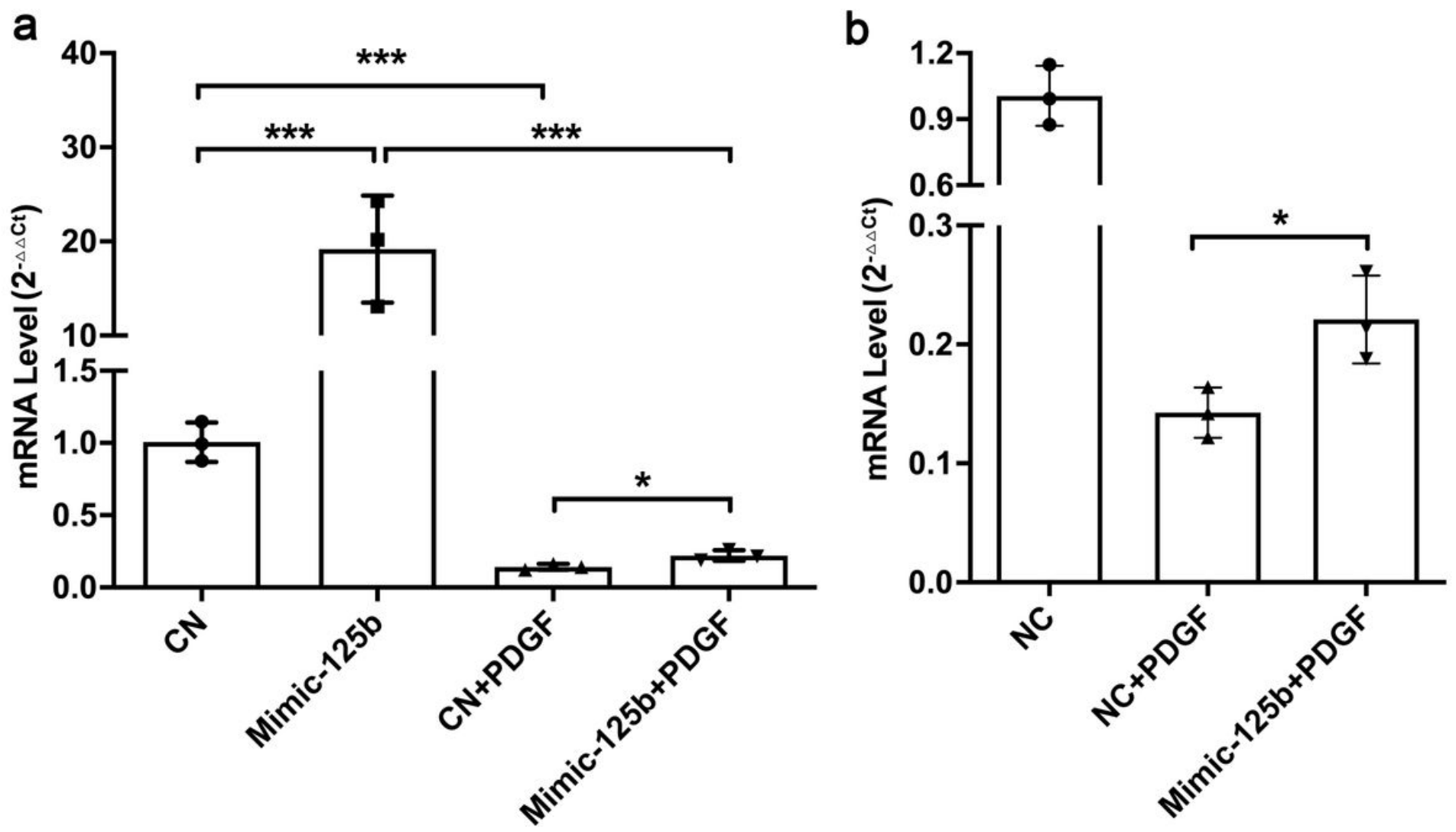

Figure 1

PDGF-BB inhibits the expression level of miR-125b in VSMC. ( $a$ and $b$ ) Vascular smooth muscle cells (A10 cell) are seeded in 6-well plates for 24h, followed by treatment of mimic-125b-loaded liposomes or mimic-NC-loaded liposomes for $24 \mathrm{~h}$ (NC or Mimic-125b group), then the two groups are treated with PDGF-BB or medium for another 24h (NC, Mimic-125b, NC+PDGF-BB and Mimic-125b+PDGF-BB groups). Finally, cells are harvested for testing the level of miR-125b by qT-PCR. The level of miR-125b is upregulated in Mimic-125b group, about 20 folds $(p<0.001)$; and which is down regulated in NC_PDGF-BB group ( 95\%, $p<0.001)$ and Mimic-125b+PDGF-BB group ( 80\%, $p<0.001)$. 

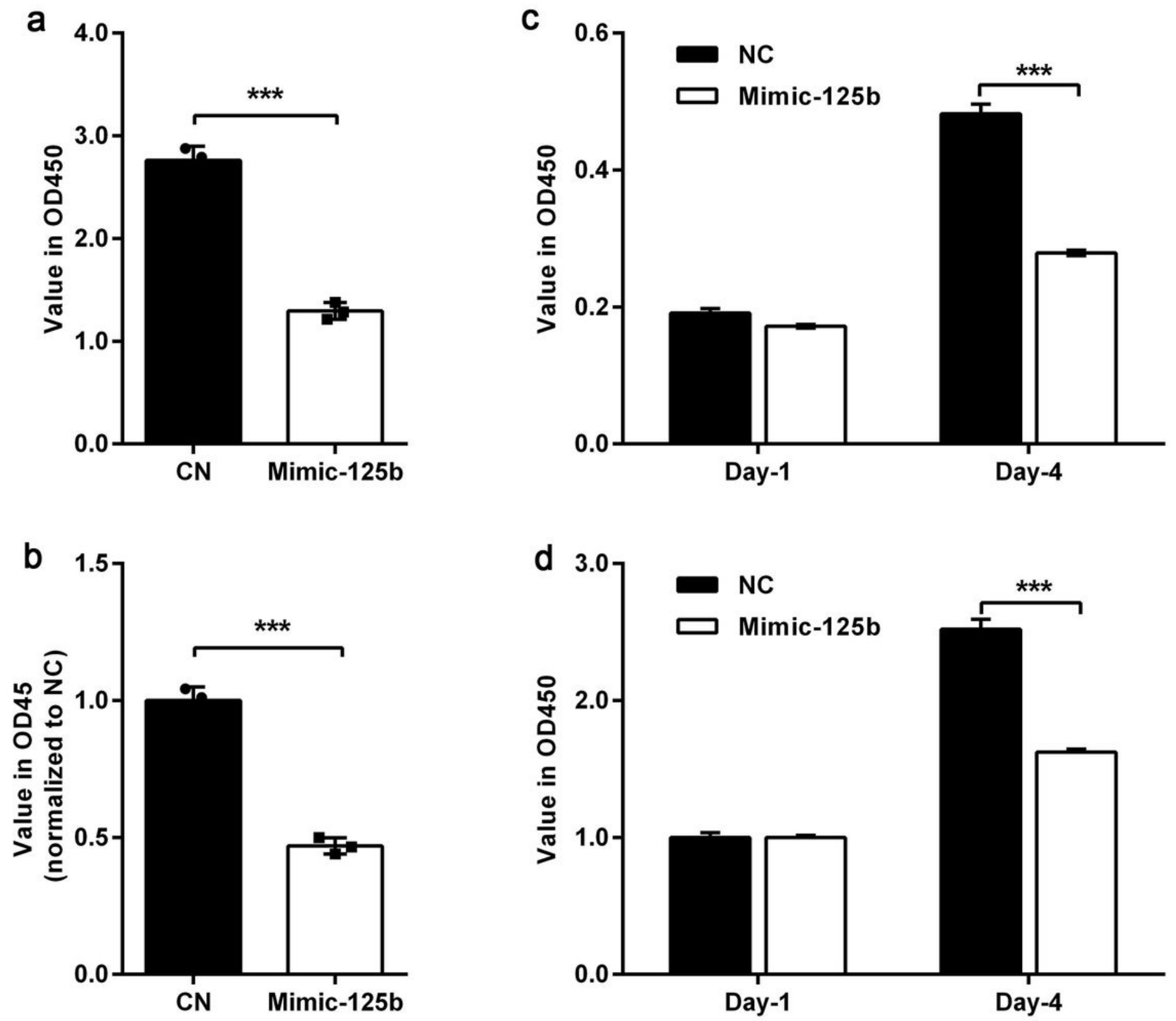

Figure 2

miR-125b restricts the cellular proliferation in AVSM cells. ( $a$ and b) 10A cells are seeded in 96-well plates for $24 \mathrm{~h}$, then treated with mimic-125b-loaded liposomes or mimic-NC-loaded liposomes for another $24 \mathrm{~h}$, which followed by 24h-treatment of PDFG-BB. Then cellular proliferation is tested by CCK8 assay. Absolute OD values in 450nm show the down-regulation of cell proliferation in Mimic-125b group $(p<0.001)$, normalized values show the down-regulation of cell proliferation in Mimic-125b group, about $50 \%(p<0.001)$. ( $c$ and $d) 10 \mathrm{~A}$ cells are seeded in 96 -well plates for $24 \mathrm{~h}$, then treated with mimic-125bloaded liposomes or mimic-NC-loaded liposomes for another 24h, which followed by $24 \mathrm{~h} / 96 \mathrm{~h}$-treatment of PDFG-BB. Then cellular proliferation is tested by Brdu assay. Absolute OD values in $450 \mathrm{~nm}$ show the 
down-regulation of cell proliferation in Mimic-125b group in day $4(p<0.001)$, normalized values show the down-regulation of cell proliferation in Mimic-125b group in day 4 , about $30 \%(p<0.001)$.
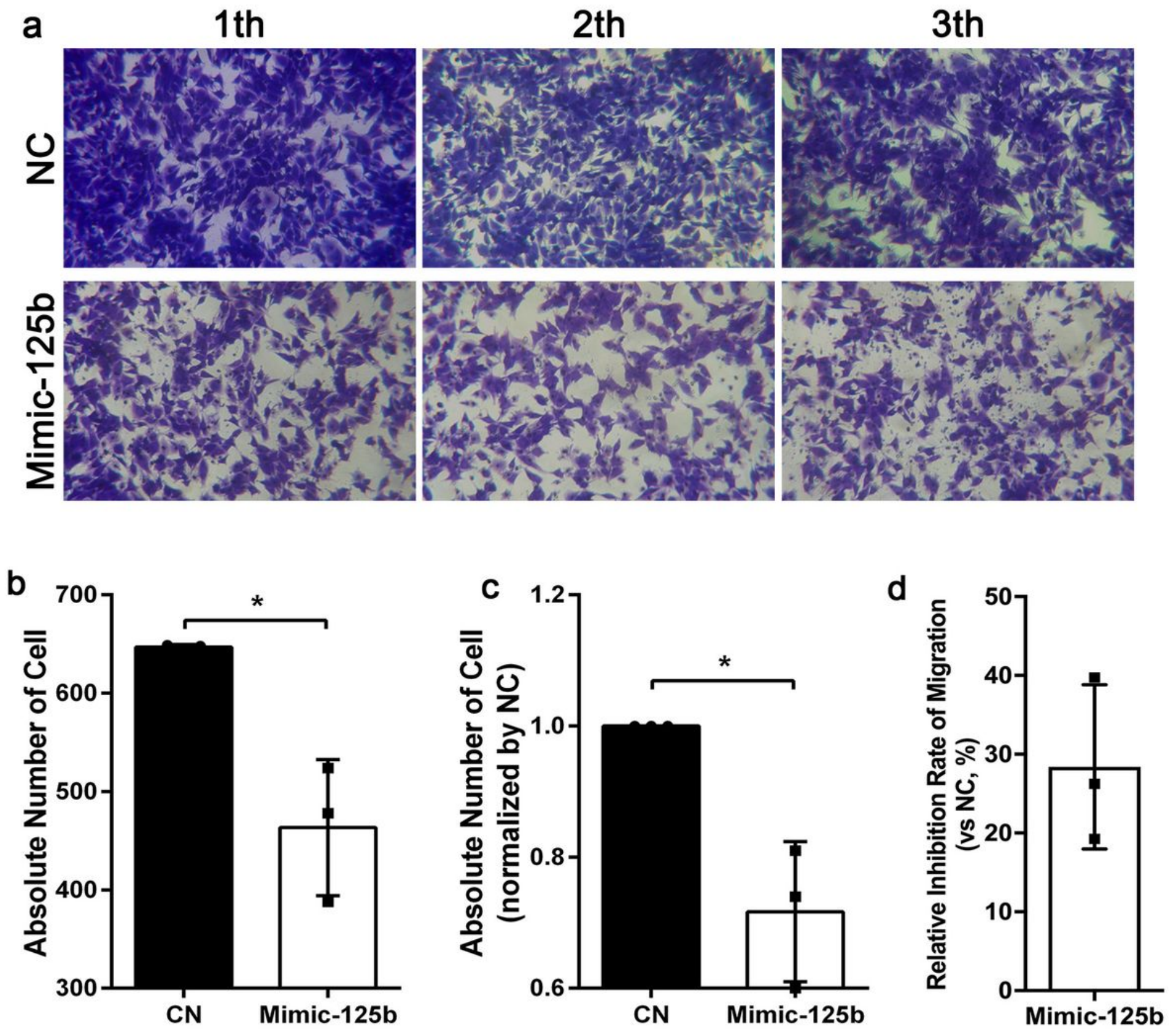

Figure 3

miR-125b inhibits the migration of VSMCs. (a) 10A cells are seeded in 6-well plates for $24 \mathrm{~h}$, then undergo 24h-treatment of mimic-125b-loaded liposomes or mimic-NC-loaded liposomes, after which cells are transplanted into trans-well for another 48h. (b) Migrated cells are calculated, and it shows the decreased migrated cells in Mimic-125b group ( $p<0.05$ ). ( $c$ and $d$ ) Normalized values show about $30 \%$ decreasing of migrated cells in Mimic-125b group $(p<0.05)$. 

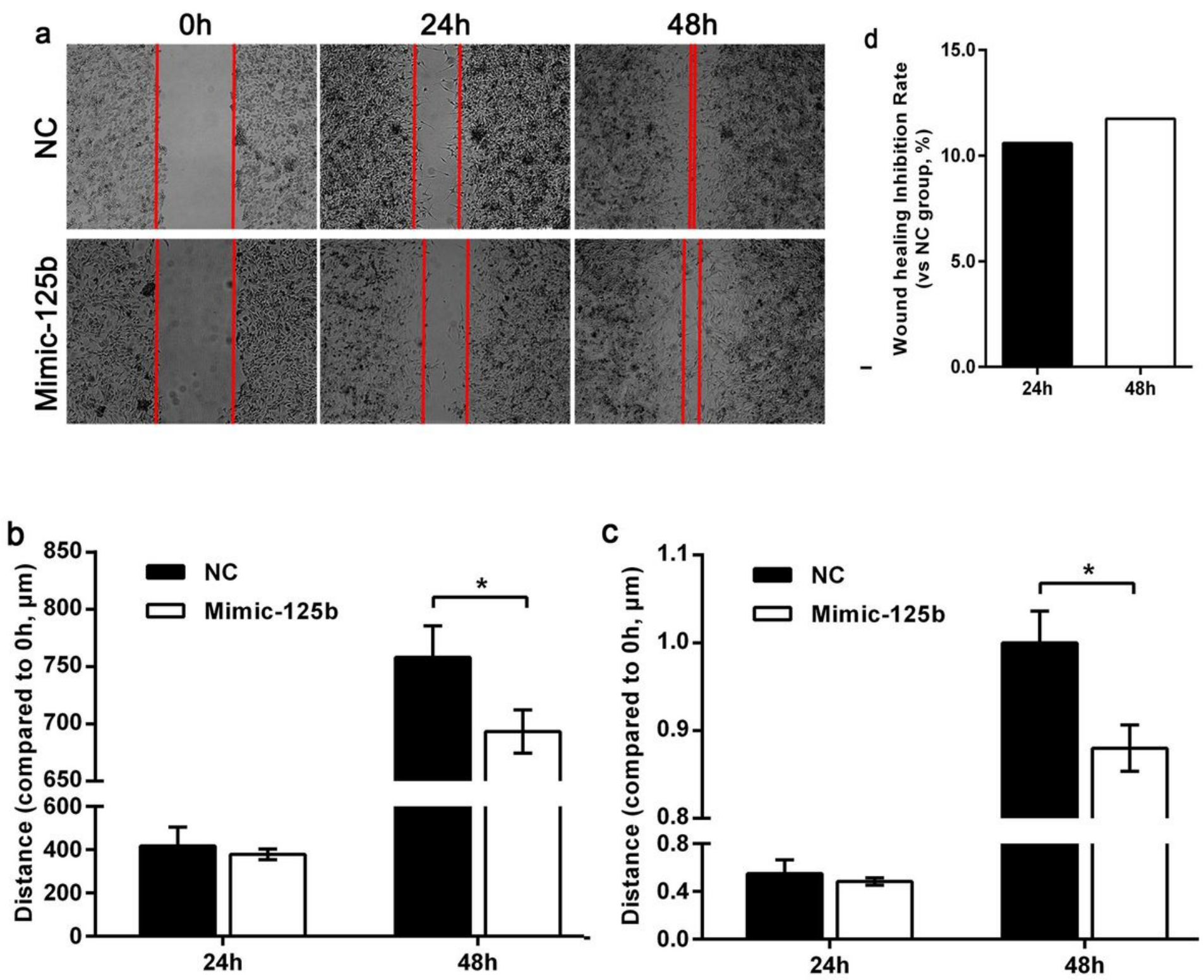

Figure 4

miR-125b inhibits the invasion of VSMCs. (a) 10A cells are seeded in 6-well plates for $24 \mathrm{~h}$, then treated with mimic-125b-loaded liposomes or mimic-NC-loaded liposomes for $24 \mathrm{~h}$, which followed by wound healing assay for another 48h. (b) Migration distance is calculated, which shows the decreasing in Mimic-125b group in day 2 ( $p<0.05$ ). (c and d) Normalized values show about $10 \%$ decreasing of migration distance in Mimic-125b group in day 1 ( $p>0.05)$, and about $12 \%$ decreasing of migration distance in Mimic-125b group in day $2(p<0.05)$. 


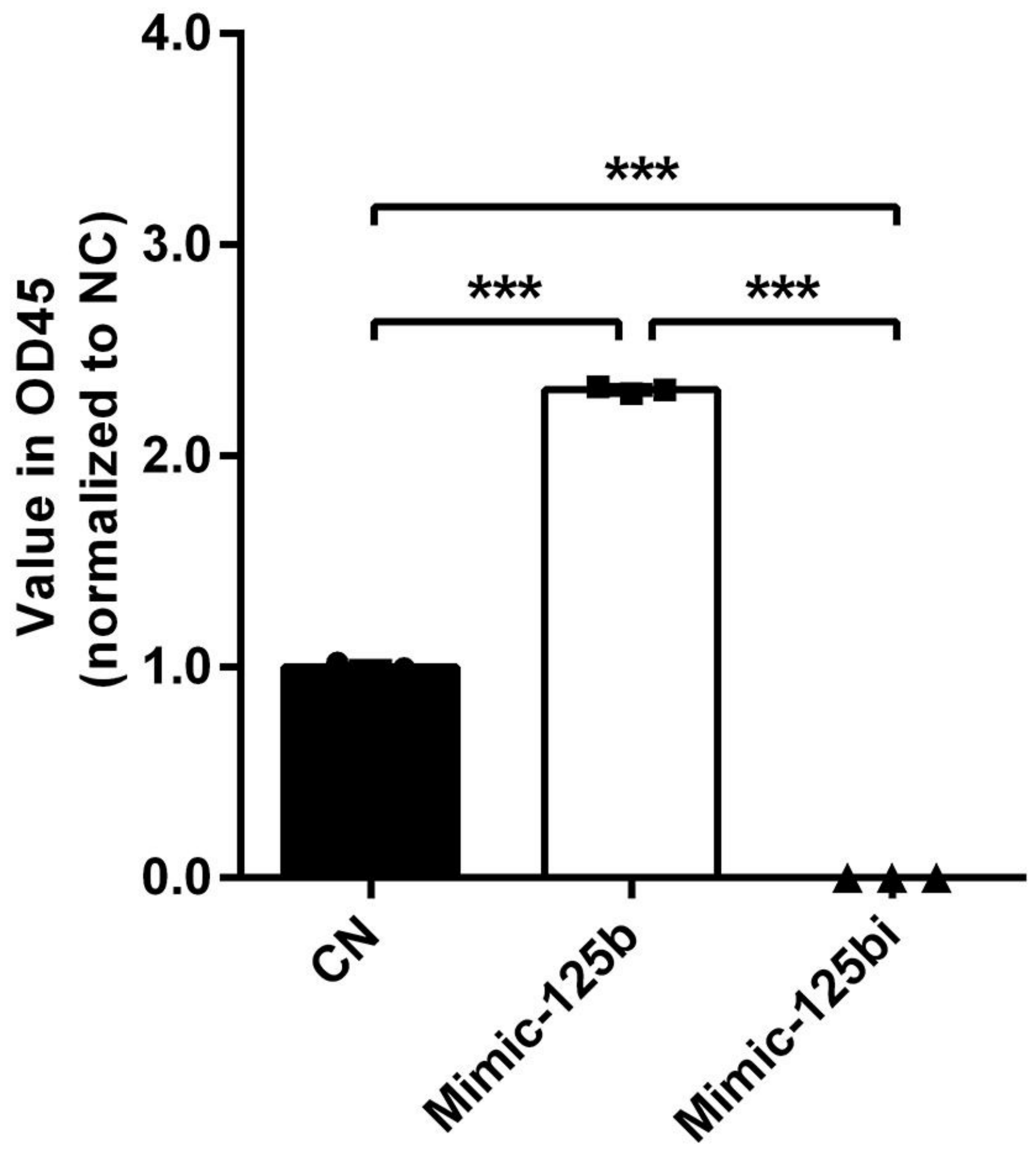

Figure 5

miR-125b is regulated by mimic-125b and mimic-125b inhibitor. $10 \mathrm{~A}$ cells are seeded in 6 -well plates for 24h, then treated with mimic-125b-loaded liposomes, mimic-NC-loaded liposomes or mimic-125b inhibitor (Mimic-125bi) for 24h, which followed by qT-PCR assay. Mimic-125b group shows about 2-fold upregulation of miR-125b ( $<<0.001)$, while the Mimic-125bi group shows about $100 \%$ down-regulation of $\operatorname{miR}-125 b(p<0.001)$. 

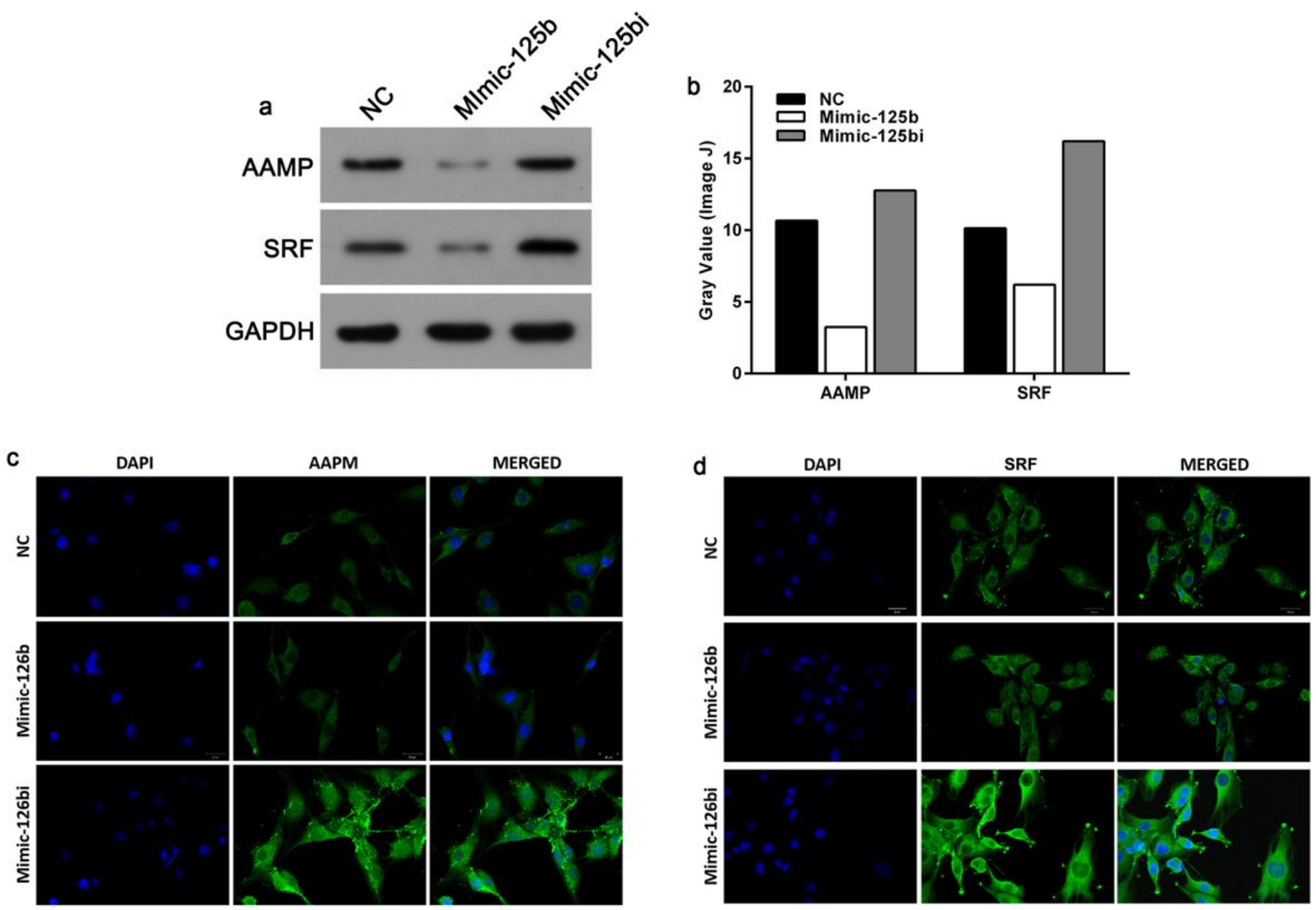

Figure 6

miR-125b plays a negative role in the regulation of AAMP and SRF. ( $a$ and b) 10A cells are seeded in 6well plates for $24 \mathrm{~h}$, and then treated with mimic-125b-loaded liposomes, mimic-NC-loaded liposomes or mimic-125b inhibitor (Mimic-125bi) for 24h, which followed by western blot assay. Mimic-125b group shows about $60 \%$ down-regulation of AAMP and about $50 \%$ down-regulation of SRF, and Mimic-125bi group shows about 1.25-fold up-regulation of AAMP and about 1.5-fold up-regulation of SRF. (c and d) Same treatments are applied in $10 \mathrm{~A}$ cells, which are followed by IF assay. 